\title{
The Tycho double star catalogue $e^{\star \star \star}$
}

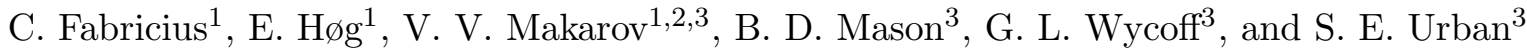 \\ 1 Copenhagen University Observatory, Juliane Maries Vej 30, 2100 Copenhagen Ø, Denmark \\ 2 Universities Space Research Association, 300 D Street S.W., Washington, DC 20024, USA \\ 3 United States Naval Observatory, 3450 Massachusetts Avenue N.W., Washington, DC 20392-5420, USA
}

Received 20 November 2001 / Accepted 18 December 2001

\begin{abstract}
We report the discovery of 13251 visual double stars, mostly with separations between 0.3 and 1 arcsec, from a dedicated re-reduction of the Tycho data from the star mapper of the ESA Hipparcos satellite. The new doubles are combined with 18160 WDS systems identified in the Tycho-2 Catalogue, and 1220 new Tycho-2 doubles, to form the Tycho Double Star Catalogue, TDSC, a catalogue of absolute astrometry and $B_{\mathrm{T}}, V_{\mathrm{T}}$ photometry for 66219 components of 32631 double and multiple star systems. We also include results for 32263 single components for systems unresolved in TDSC, and a supplement gives Hipparcos and Tycho-1 data for 4777 additional components. The TDSC thus contains a total of 103259 entries. Cross identifications are given to WDS, HD, Hipparcos and Tycho-2.
\end{abstract}

Key words. stars: binaries: visual - catalogs - astrometry

\section{Introduction}

For centuries, visual double stars have been studied with the goal of determining stellar masses and the statistics for binary and multiple star systems. Traditionally, such systems have been studied visually using a micrometer on a long-focus instrument. In this way separations and position angles can be measured with high precision. In more recent work (e.g., Mason et al. 2000a) speckle interferometry has successfully replaced the eyepiece micrometer.

The Washington Double Star Catalog, WDS, (Mason et al. 2001b) is the official IAU double star data base. At the time when we initiated the present project (July 2000), it contained 83287 pairs of stars. Due to the nature of the catalogue, it gives only approximate coordinates; the RA, e.g., is given with a resolution of $0.1 \mathrm{~min}$. This can lead to difficulties in identifying the systems or individual components unambiguously in other catalogues and on the sky. It is therefore desirable to provide accurate absolute coordinates for each component of a system. Accurate positions are also important for

\footnotetext{
Send offprint requests to: C. Fabricius,

e-mail: cf@astro.ku.dk

* Based on observations made with the ESA Hipparcos astrometry satellite.

$\star \star$ The catalogue is only available in electronic form at the CDS via anonymous ftp to

cdsarc.u-strasbg.fr $(130.79 .128 .5)$ or via

http://cdsweb.u-strasbg.fr/cgi-bin/qcat?J/A+A/384/180
}

determining the mass ratio in orbiting pairs and for understanding the nature of a system, because proper motions can often tell a physical component from an optical.

The smaller Catalogue des Composantes d'étoiles Doubles et Multiples, CCDM (Dommanget \& Nys 1994) gives more accurate coordinates, but contains only 34031 systems, and we have therefore not used it in the present work. A new version of the CCDM is being prepared (Dommanget 2000), but will not include Tycho results.

The Hipparcos Catalogue (ESA 1997) includes an annex of components of double and multiple systems with very accurate astrometry for 12195 systems of which about 3000 were new systems.

The Tycho-2 Catalogue (Høg et al. 2000b), contains no double star annex, but in an analysis of the Tycho-2 double star solutions, Mason et al. (2000b) found 1234 new systems, which were included in the WDS (Mason et al. 2001b), but 14 of these are left out of the Tycho Double Star Catalogue, TDSC, because they were found very uncertain. Also many known systems were extracted from the Tycho-2 Catalogue for insertion into the WDS. As described in Mason et al. (2000b) these came from two sources:

- 7485 Tycho-2 double star measures result from a special processing called "double star treatment" (Col. 201 of the Tycho-2 Catalogue); 
- 5283 double stars come from more widely separated systems given a "normal treatment".

The identification of doubles in Tycho- 2 was based on a rather narrow, but safe, search window and several thousand Tycho-2 doubles were left behind.

When the Tycho-2 Catalogue was prepared, the rather slow duplicity analysis was only carried out for a small subset of the stars, suspected to be non-single. We have now looked at all Tycho-2 stars and resolved 17000 more systems, including about 13000 discoveries. The large number of new systems has been reached not only by analysing more stars, but also through allowing solutions with smaller separations than the limit of 0.8 arcsec used in Tycho-2. It should be noted that for the smallest separations, the risk of spurious detections or large errors in $\rho, \theta$ is increased.

Having noticed that the identification of double stars is often difficult, and that the WDS at the time did not include all Tycho results, we decided to make the Tycho-2 doubles more easily accessible. We have therefore used this opportunity to include in the present TDSC, not only the newly discovered systems, but also the doubles already in the Tycho-2 Catalogue. All double star information from the TDSC will be included in the WDS.

For most double stars it takes quite a few measures spanning several years to classify them as having motion which is either Keplerian or rectilinear; typically ten is the minimum. Of the systems observed by Tycho, 13800 of them have between two and nine measures, other than the Tycho measure. For these, the Tycho measure will aid in eventual motion characterization. Tycho also made measures of 6441 systems having between 10 and 50 historical observations. It is usually these which would be considered as the "ripest" for orbit analysis. Of the (now) 84486 WDS systems, only 1430 (less than 2\%) have orbit determinations. Work on this continues. A small number (1509) of the Tycho double star measures are of systems which are well characterized (i.e., number of measures greater than 50). Many of these are quite useful in giving an independent assessment of Tycho double star measures.

\section{The Tycho catalogues}

The observations from the Tycho experiment onboard the Hipparcos satellite were exploited to produce the Tycho-1 Catalogue (ESA 1997) of one million stars with positions and $B_{\mathrm{T}}, V_{\mathrm{T}}$ magnitudes. Many doubles with separations above 2 arcsec were resolved.

The Tycho-2 Catalogue (Høg et al. 2000b), which has superceded Tycho-1, is the result of a second data reduction, using more advanced and demanding techniques. The Tycho-2 Catalogue contains positions, proper motions and $B_{\mathrm{T}}, V_{\mathrm{T}}$ magnitudes for 2.5 million stars. The proper motions were derived from the Tycho- 2 positions, the 100-year-old positions in the Astrographic Catalogue and 143 other astrometric catalogues.
A supplement to Tycho-2 contains stars from Tycho-1 and Hipparcos, including double star components, which are not in Tycho-2. Close Hipparcos doubles which are included, but unresolved, in Tycho-2 are, however, not part of the supplement. For completeness and to reduce ambiguities, we have included data from this supplement in the present work when cross referencing to the WDS, and the relevant entries are given in the TDSC Supplement. A second supplement to Tycho- 2 contains Tycho-1 stars, also not in Tycho-2, which are suspected to be either ghosts produced by sidelobes or heavily disturbed detections. Here we have ignored this second supplement.

In Tycho-2, double stars with separations down to 0.8 arcsec were resolved in a dedicated double-star reduction process, carried out for only a small subset of the stars. The production of the Tycho-2 Catalogue was based on an input catalogue, allowing a field of radius 2.5 arcsec around each input position to be analysed. For the dedicated double star analysis to work, both components must be within this field. This sets an upper limit to the separation unless the components have separate entries in the input catalogue.

Now, a third catalogue, the Tycho Double Star Catalogue, complements Tycho-2 with respect to double stars. It presents accurate positions, proper motions, $B_{\mathrm{T}}$ and $V_{\mathrm{T}}$ photometry for 66219 components of 32631 visual double and multiple star systems, as well as results for 32263 systems, unresolved by Tycho. In contrast to the system based WDS, where pairs of stars are listed, we give, as far as possible, results for the individual components, with one line per star. For 4777 additional components, mostly faint, which were not detected in Tycho-2, we give a supplement with the Hipparcos or Tycho-1 positions, including the Hipparcos double star components, copied across from the Tycho- 2 supplement. The TDSC contains a total of 103259 entries, including the supplement. The source of astrometry is indicated for all stars by a flag.

Observations spread over 3 years are combined to yield mean astrometry for that period, allowing only for the (linear) proper motion known in the input catalogue. Any additional motion will cause smearing of the image, and doubles with a short orbital period will therefore not be detected.

Due to the nature of the Tycho data, only systems with modest magnitude differences can be detected. As is shown in Fig. 1, it rarely exceeds 2 mag. Most systems have primaries of about $V_{\mathrm{T}}=11.5 \mathrm{mag}$, and seldom fainter than $12 \mathrm{mag}$. For separations below 1 arcsec, the conditions are difficult, and the limit is brighter. It may also be noticed that we have the best conditions for separations between 1 and 3 arcsec. This is due to the size of the input fields in the Tycho- 2 data base. The Tycho separations go from 0.29 arcsec to 4680 arcsec. The larger separations are based on normal treatment of two separate input fields. The supplement also contains smaller separations, down to 0.13 arcsec, for pairs involving a Tycho star and a (faint) Hipparcos star. 


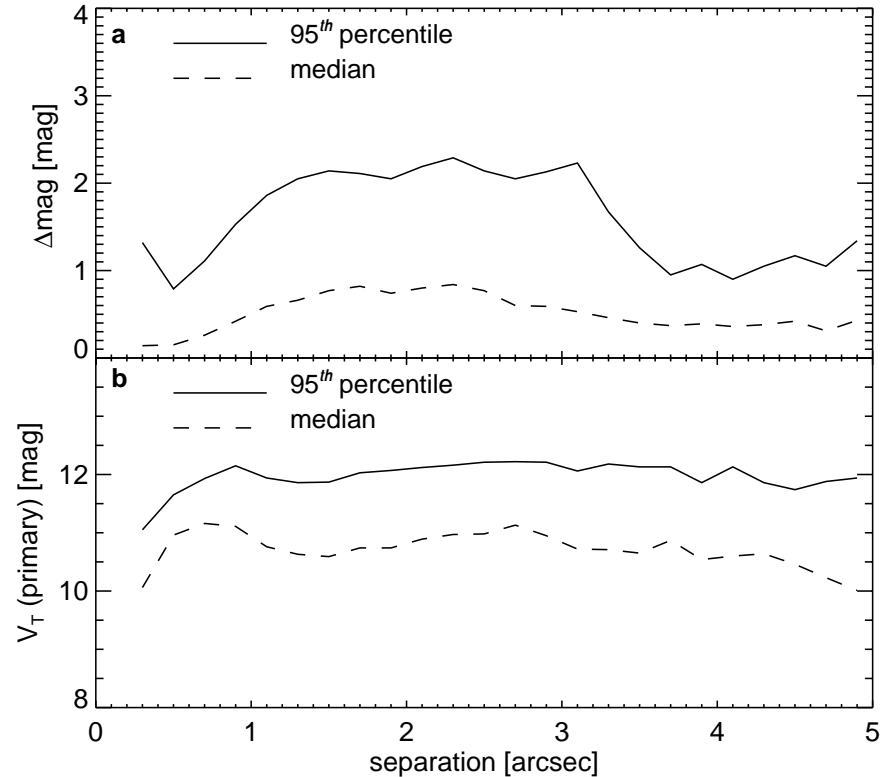

Fig. 1. The distribution of magnitude difference and actual magnitude is indicated for the smaller separations.

\section{Stellar contents of the TDSC}

In the Tycho Double Star Catalogue, we have included three groups of stars:

- Tycho double star solutions;

- Tycho-2 stars identified in WDS;

- Tycho-2 pairs separated by less than 10 arcsec.

\subsection{Tycho double star solutions}

New double star solutions were attempted for all Tycho-2 stars, looking for a companion within the field of 2.5 arcsec radius. The method used for Tycho-2, as described in $\mathrm{H} \varnothing \mathrm{g}$ et al. (2000a), was adopted with a few modifications. First of all, stars which did not look promising after a few iterations were deemed single and no further analyses were attempted. This was necessary to save computing time, but some close doubles may have been lost. Secondly, new criteria were introduced regarding when to terminate the loop of iterations, instead of the fixed number of iterations in Tycho-2. This was partly introduced for efficiency and partly because some systems were converging very slowly. As a consequence there can be minor discrepancies between the new solutions and the original Tycho- 2 solutions. Finally, the criteria, regarding which solutions to accept and which to discard, were revised. Smaller separations than the very conservative limit of 0.8 arcsec used in Tycho-2 were accepted, but at the same time a separation dependent limit on the signal-to-noise ratio was introduced. The smallest accepted separation in the Tycho solutions was 0.29 arcsec.

A few stars which were excluded from Tycho-2 have now been successfully resolved, and accepted for the present catalogue. They are the pairs TDSC 28625, 42517 and 51915 .

\subsection{Identification of Tycho-2 stars in WDS}

A cross reference list between WDS and Tycho-2 was presented by Mason et al. (2000b). It was, however, soon realised that a more complete, but perhaps less safe, list should be produced. This new list should not only list the primary components, but also sort out the individual components of multiple systems.

The WDS, being compiled from hundreds of sources, is very inhomogeneous and there are occasional ambiguities regarding what pairs constitute one system, or how to designate some individual components. We adopted, as a main rule, the definition that all pairs having the same position field in WDS constitute one system. For each such system, we looked up all stars in Tycho-2 (including the supplement) within a radius given by the maximum separation in the system plus 1.5 or 3 arcmin, depending on the quality of the WDS astrometry. We then looked for pairs of Tycho-2 stars which had the right separation and position angle (except perhaps for $\pm 180^{\circ}$ ) at the epoch in question. For the majority of systems, this worked fine, but a significant fraction of the cases could not be handled automatically. Often, the designations were inconsistent; or a very bright double was apparently not in Tycho-2 at all; or the same Tycho-2 star appeared in more than one system. All in all, we identified more than 48000 components in more than 23000 systems with separations between 0.13 and 4680 arcsec. These numbers include 4000 new Tycho pairs and also 4800 Hipparcos and Tycho-1 components, copied across from the Tycho- 2 supplement.

In cross referencing Tycho- 2 with WDS, there were almost 29000 systems where we found only one Tycho- 2 star in the vicinity. This Tycho- 2 star is then a likely identification for typically either the A component or the photocentre of $\mathrm{A}$ and $\mathrm{B}$. We have included these stars in the TDSC.

\subsection{Tycho-2 doubles missing in WDS}

The last group of stars consists of Tycho- 2 stars which were not found in the version of WDS we used, and which are not part of a double star solution, but which have a partner in Tycho- 2 within 10 arcsec. There were 359 such doubles, some of which have recently been included in WDS from the Washington Fundamental Catalog. The distance of 10 arcsec was chosen because doubles closer than this limit are often not resolved in scans of Schmidt plates.

\subsection{The TDSC supplement}

The TDSC Supplement contains Hipparcos and Tycho-1 data for 4777, mostly faint, resolved or unresolved components of WDS systems, which were not detected in Tycho-2. Normally, these additional components complement single components in the main TDSC. Only 316 systems in the supplement have no components in the main catalogue. 


\subsection{Incorporation into the Washington Double Star Catalog}

Following the new Tycho solutions and the new cross identification list, an additional 25235 measures (i.e. pairs), of which 13080 are resolved here for the first time, were incorporated into the WDS. The WDS is continuously growing, and at the time of inclusion of the TDSC results, a few of our discoveries were no longer discoveries. All TDSC systems have a running number, 1 . . 65210 , in the TDSC. The subset of new systems have also a discovery designation assigned, when added to the WDS. The assignment of TDS discovery designations for the new systems begins where Mason et al. (2000b) leaves off, i.e., TDS 1235. The four-digit discovery number has been adequate for all historical binary star work. However, the work here exceeds this. For the WDS we adopt the admittedly somewhat makeshift strategy where all TDS numbers beyond 9999 are assigned to the discovery designation "TDT", beginning with "TDT 0001". Of the 12155 measures of known systems, 891 are measures where the position angle differed by 30 degrees or more, or the separation differed by $30 \%$ when compared with more recent measures. The majority of these systems seem to have small separations. Of those with larger separations many do not have contemporary measures, so the Tycho-2 measure may not reflect an error, simply a difference. The relevant components of these 891 systems are flagged in the catalogue.

\section{Construction of the catalogue}

The construction of the TDSC follows the same main principles as for Tycho-2, as described in Høg et al. (2000a), and uses the same astrometric and photometric calibrations. Here we restrict ourselves to TDSC specific questions.

We should, however, mention the problem of sidelobes. Due to the design of the Tycho instrument, with eight long slits (see ESA 1997, for details), sidelobes will cause a disturbance at characteristic distances from each star. This gives a risk of spurious detections of "new" components or double stars, due to the sidelobes. We have checked relevant cases in an attempt to eliminate such mistakes.

\subsection{Relative positions}

In addition to the absolute positions given for each component, we also derive the traditional relative positions in terms of position angle and separation. Starting from the first component, the entire geometry of the system can easily be established from the relative positions only.

In the Hipparcos and Tycho Catalogues, the observed position for a star results from the combination of a large number of one-dimensional observations along different directions. This mean position therefore represents a slightly different epoch in right ascension and declination, and these epochs will differ from one star to the next, though only slightly for neighbouring stars. The separations and
Table 1. Distribution of number of components in each system, for the main TDSC catalogue, for new systems in the main catalogue, and for the supplement.

\begin{tabular}{rrrrr}
\hline $\begin{array}{c}\text { N } \\
\text { comp }\end{array}$ & $\begin{array}{c}\text { main } \\
\text { catalogue }\end{array}$ & $\begin{array}{c}\text { new } \\
\text { systems }\end{array}$ & $\begin{array}{c}\text { supplement } \\
\text { catalogue }\end{array}$ & total \\
\hline 1 & 32263 & - & 4420 & 28572 \\
2 & 31785 & 13250 & 166 & 35432 \\
3 & 758 & 1 & 7 & 1047 \\
4 & 76 & & 1 & 132 \\
5 & 7 & & & 16 \\
6 & 3 & & & 8 \\
7 & 1 & & & 1 \\
8 & 0 & & & 1 \\
11 & 1 & & & \\
\hline
\end{tabular}

position angles we present here are computed for an epoch which is the mean of the four relevant epochs. As in the Hipparcos Catalogue, the position angles are given with respect to the ICRS pole. This is unlike traditional double star observations which are made relative to the pole of observation date. Standard errors of position angle and separation have been derived, taking the correlations between RA and Dec into account.

\subsection{Photometry}

The photometry in the catalogue is either copied across from Tycho-2 or new TDSC photometry, processed using the same set of calibrations as for Tycho-2. For the supplementary Hipparcos and Tycho-1 stars, which were copied across to the TDSC Supplement for completeness, the situation is more complex. For about half of them, $B_{\mathrm{T}}, V_{\mathrm{T}}$ photometry is taken from Tycho- 1 or from Fabricius \& Makarov (2000a). For the other half, only the Hipparcos magnitude, $H p$, is given. A flag in the catalogue specifies the origin of the photometry.

\subsection{TDSC identification}

The stars in TDSC are identified by a running number which specifies the system, and a one- or two-character component designation.

The number of components in each system is shown in Table 1. Multiple systems in TDSC may well consist of more than one physical system. We have only looked at the WDS position to define the number of members in a system. E.g., a "triple" system like TDSC 232 (WDS $00059+1805$ ) consists of an A-B pair with high proper motion (200 mas/yr) and a C component at a large separation and with small proper motion. Nothing indicates a physical connection between the $\mathrm{C}$ component and the $\mathrm{A}-\mathrm{B}$ pair.

\subsection{Component reversal}

In the double star processing, the signal from one of the components is subtracted from the total signal at the 

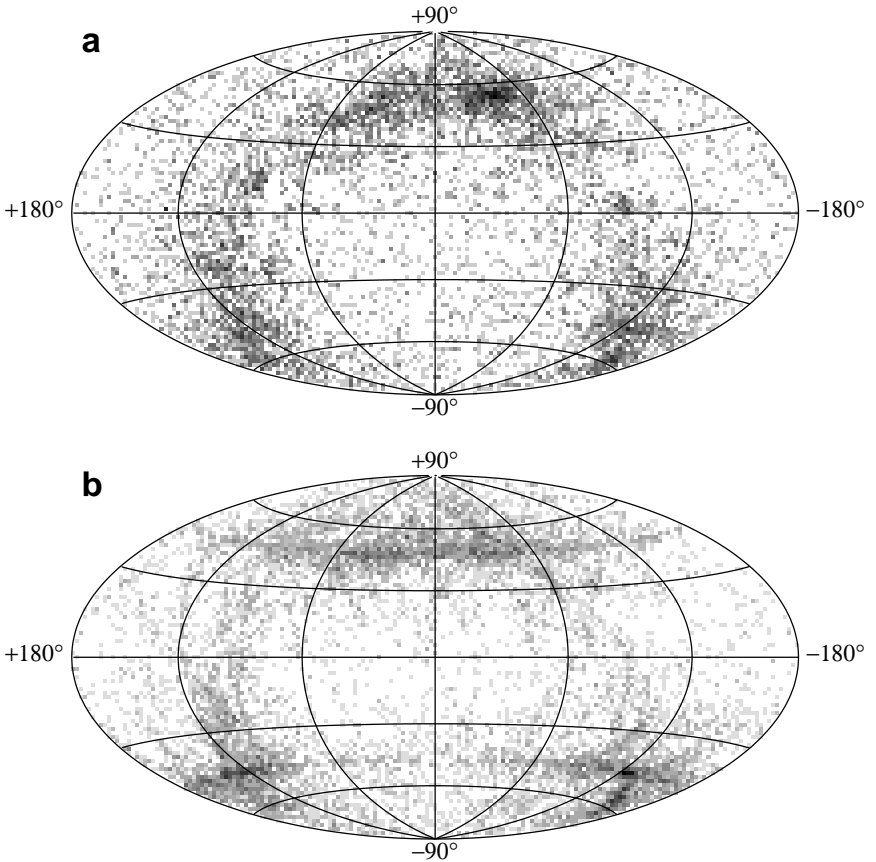

Fig. 2. The sky distribution in ecliptic coordinates of TDSC systems known in the present WDS (panel a)), and newly discovered systems b).

assumed location of that component and with the assumed amplitudes, and the other component is then sought in the residual signal. The process is then repeated, but now the second component is subtracted and an improved position for the first is determined. As many iterations are made as required to obtain a stable solution. Unfortunately, a stable solution does not always mean a correct solution. Examples are known for close doubles, where we have found the bright component near the photocentre, leaving a rather strong residual signal on the opposite side of the primary. The secondary is then found with an error of $180^{\circ}$ in position angle. Such erroneous solutions with "component reversal" can be quite stable, and fulfill the convergence criterion.

For all relevant solutions, we computed alternative solutions assuming that the first suffered from component reversal. We then restarted the iterations, but starting from the alternative solution. If the second set of iterations converged to a different solution, the signal-to-noise ratios and the separation values were used to judge which was the correct solution. Despite this effort, we cannot exclude that a number of "reversed" solutions have survived.

\subsection{Notes}

Notes are provided for some stars to explain peculiarities, particularly related to the identification. The value of the note flag indicates which kind of problem has been found. We distinguish between unclear or ambiguous WDS designations, WDS components we have apparently resolved, stars added to a WDS system and just general notes.

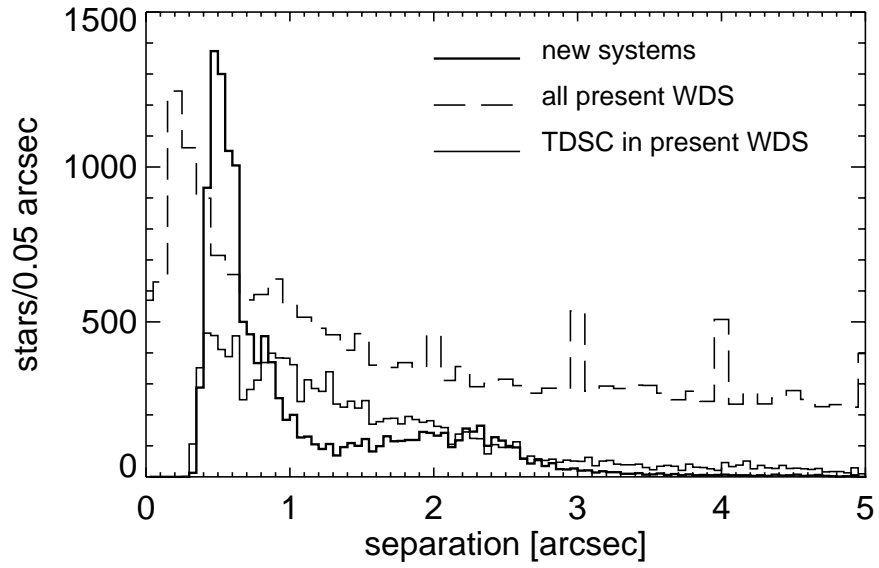

Fig. 3. The distribution of separations for all present WDS systems (dashed line), for TDSC systems known in WDS (thin line) and for new systems (thick line). Only the smaller separations are shown.

The flag for resolved WDS components should also be seen as a warning that the new component may be spurious.

\section{Evaluation of the catalogue}

An interesting property of the catalogue follows from Fig. 2, which shows the sky distribution of the 20000 known and 13000 new systems in ecliptic coordinates. The known systems have a concentration towards the Galactic plane, as could be expected, while the new systems in addition show a concentration towards ecliptic latitude $\pm 47^{\circ}$, which is only barely visible for the known systems. Due to the nature of the Hipparcos scanning law (ESA 1997, vol. 1, Fig. 3.3.3), these latitudes received more than twice the average number of observations and can therefore provide a much better signal-to-noise ratio than the more sparsely observed ecliptic region. We may conclude that a uniform survey would have discovered many more new systems.

The distribution of separations for known and new systems in TDSC is shown in Fig. 3, and the distribution for WDS systems (irrespective of magnitude) is included for comparison. For separations around 0.5 arcsec, the majority of the observed systems are new and outnumber the present WDS. The new systems mostly have separations between 0.4 and 1 arcsec, but there is a substantial number also around 2 arcsec. For separations between 0.4 and 2.3 arcsec we have observed the majority of the WDS systems.

\subsection{Astrometric comparisons}

In Fig. 4 we show a comparison of the relative astrometry between Hipparcos and the new Tycho solutions for 2699 double stars. The scatter increases toward smaller separations especially for the separation (panel a). For measured separations below $0.4-0.5$ arcsec, as indicated by the line, this is particularly pronounced. 


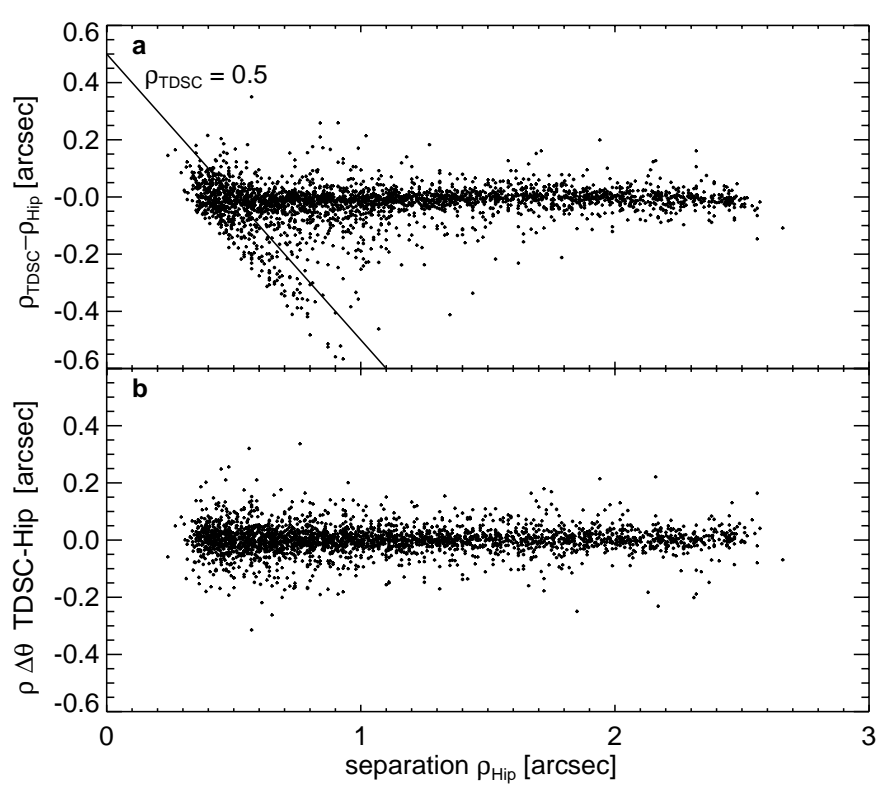

Fig. 4. Comparison of the relative astrometry between Hipparcos and new Tycho results for 2699 double stars.

As an assessment of the quality of double star measures from Tycho-2, they have been compared to systems with calibration quality orbits (see the 5 th Orbit Catalog, Hartkopf et al. 2001). While the orbit catalog contains many definitive orbits it also contains a like number of wider, long-period systems whose orbits, while describing the complete motion inexactly, provide perfectly reasonable ephemerides over the observation dates. Two examples of these are given in Figs. 5 and 6 . The full list of residuals to calibration systems is presented in Table 2 . The first four columns identify the system by providing the TDSC running number, the epoch 2000 coordinate, discovery designation, and Hipparcos number. All of these systems have a significant observational history, and these tend to be quite bright. Therefore, all of these systems have Hipparcos numbers. One might reasonably wonder how these Tycho-2 observations compare for the fainter systems (i.e., those with no Hipparcos number), however, that sort of comparison is not possible here, since typically only brighter systems have enough observations to be deemed "calibration quality". Columns five through seven give the epoch of observation, the position angle (in degrees), and the separation (in seconds of arc). Columns eight and nine give the $\mathrm{O}-\mathrm{C}$ orbit residuals (in $\theta$ and $\rho$ ) to the orbit referenced in column ten. The systematic overestimating of separations in very close systems which has been noted for other techniques (Worley 1981) is perhaps also seen here, where the mean for the 18 close systems (i.e., $\rho<1^{\prime \prime}$. 0$)$ is $\left(\mathrm{O}-\mathrm{C}_{\rho}\right)=0$.'02. The mean $\frac{\left|\left(\mathrm{O}-\mathrm{C}_{\rho}\right)\right|}{\rho}=$ $1.42 \%$, which compares quite well with ground-based high angular resolution work ${ }^{1}$. While close systems are measured, at separations less than about 1 arcsec the error

\footnotetext{
1 Mason et al. (2001a) find $\frac{\left|\left(\mathrm{O}-\mathrm{C}_{\rho}\right)\right|}{\rho}=1.1 \%$ for speckle interferometry with the USNO 26 inch telescope.
}

can be considerable; as shown in Fig. 7. While these measures with larger errors are still of value in systems with few measures, their value in systems with many measures (especially those with contemporaneous double star observations) is more limited.

\subsection{Photometric comparisons}

For close Hipparcos doubles, $B_{\mathrm{T}}, V_{\mathrm{T}}$ photometry is given by Fabricius \& Makarov (2000a), (FM for short). They have analysed the Tycho data, taking advantage of the very accurate Hipparcos astrometry to obtain Tycho photometry for 9473 components of 5173 systems. The separations are between 0.3 and 2.5 arcsec. In Fig. 8 we compare this special photometry with the TDSC photometry. The fainter stars are generally brighter in TDSC than in FM by some 0.1 , and the effect is more pronounced for $\mathrm{B}$ components than for A components. A few per cent of the stars, mostly B components, show large differences of 0.25 or more. The faint $\mathrm{B}$ components get brighter and the bright $\mathrm{B}$ components fainter; for the A component it is the reverse. The colours are better behaved. The faint stars are perhaps 0.05 redder in TDSC than in FM, but the number of outliers is quite modest. Figure 9 shows the distribution of the more than 5000 components in the comparison, with respect to magnitude and separation. The upper panel shows the distribution of stars with modest errors and the bottom panel shows the ones with larger errors. Large deviations occur almost entirely for small separations, and what is to be considered "small" depends on the magnitude.

\subsection{Hipparcos single stars resolved in TDSC}

Somewhat unexpectedly, six of the TDSC doubles turned out to be unresolved in the Hipparcos Catalogue. The immediate worry, that they must be false detection and indicate a low reliability for TDSC, is fortunately unwarranted. Three of the stars, HIP 14388, 47053A and 93519 are already known as doubles in the WDS. For the other three, HIP 25085, 86869 and 98334, we tried to make new Hipparcos solutions from the published transit data using the same method as in Fabricius \& Makarov (2000b). All three solutions confirm the TDSC relative positions, but they are at the same time suffering from various problems. For HIP 25085 we get a well behaved Hipparcos solution with a separation of 0.66 arcsec, but the magnitude difference is almost $4 \mathrm{mag}$, as compared to $0.2 \mathrm{mag}$ in TDSC. One may speculate that the Hipparcos solution is weakened by a separation close to half the grid period. For HIP 86869, the new solution gives about the same magnitudes as in TDSC, but a proper motion of almost 300 mas/year for the fainter component, which must then be a foreground star. Finally, HIP 98334 is in fact a single star, but has been playing us an odd trick, possibly because it is a Mira variable. The corresponding TDSC entries have been removed. 
Table 2. Tycho measures of calibration quality orbits.

\begin{tabular}{|c|c|c|c|c|c|c|c|c|c|c|}
\hline TDSC & $\begin{array}{c}\text { WDS or } \\
\alpha, \delta(2000)\end{array}$ & $\begin{array}{r}\text { Discc } \\
\text { desigr }\end{array}$ & $\begin{array}{l}\text { very } \\
\text { ation }\end{array}$ & HIP & $\begin{array}{c}\text { Epoch } \\
1990 .+\end{array}$ & $\begin{array}{c}\theta \\
\left({ }^{\circ}\right)\end{array}$ & $\begin{array}{c}\rho \\
\left({ }^{\prime \prime}\right)\end{array}$ & $\begin{array}{c}\mathrm{O}-\mathrm{C}_{\theta} \\
\left({ }^{\circ}\right)\end{array}$ & $\begin{array}{c}\mathrm{O}-\mathrm{C}_{\rho} \\
\left({ }^{\prime \prime}\right)\end{array}$ & $\begin{array}{c}\text { Orbit } \\
\text { Reference }\end{array}$ \\
\hline $227{\mathrm{~A}-\mathrm{B}^{1}}^{1}$ & $00057+4549$ & STT & 547 & 473 & 1.70 & 179.2 & 6.06 & 0.7 & 0.09 & Popović \& Pavlović (1996) \\
\hline $227{\mathrm{~A}-\mathrm{B}^{1}}^{1}$ & $00057+4549$ & $\mathrm{STT}$ & 547 & 473 & 1.70 & 179.2 & 6.06 & 0.7 & 0.01 & Heintz (1993) \\
\hline $1552 \mathrm{~A}-\mathrm{B}$ & $00373-2446$ & $\mathrm{BU}$ & 395 & 2941 & 1.74 & 295.2 & 0.71 & 1.1 & -0.01 & Pourbaix (2000) \\
\hline $3804 \mathrm{~A}-\mathrm{B}$ & $01398-5612$ & DUN & 5 & 7751 & 1.71 & 192.6 & 11.336 & 0.1 & -0.043 & van Albada (1957) \\
\hline $4630 \mathrm{~A}-\mathrm{B}$ & $02020+0246$ & $\mathrm{STF}$ & 202 & 9487 & 1.82 & 278.9 & 1.851 & 1.8 & -0.023 & Scardia (1983a) \\
\hline 9238 A-B & $04233+1123$ & $\mathrm{STF}$ & 535 & 20472 & 1.70 & 286.6 & 1.211 & 1.0 & 0.027 & Hartkopf \& Mason (2000) \\
\hline $10552 \mathrm{~A}-\mathrm{B}$ & $05005+0506$ & $\mathrm{STT}$ & 93 & 23277 & 1.55 & 246.7 & 1.281 & 0.7 & 0.039 & Seymour \& Mason (1999) \\
\hline $12260 \mathrm{~A}-\mathrm{B}$ & $05364+2200$ & STF & 742 & 26328 & 1.56 & 92.0 & 3.828 & -0.6 & -0.229 & Hopmann (1973) \\
\hline $18812 \mathrm{~A}-\mathrm{B}$ & $07176+0918$ & $\mathrm{STT}$ & 170 & 35310 & 1.81 & 77.5 & 0.72 & 1.7 & 0.00 & Heintz (2001) \\
\hline 20553 A-B & $07417+0942$ & STF & 1130 & 37484 & 1.92 & 359.3 & 0.33 & 2.6 & -0.04 & Mason et al. (1999) \\
\hline 20892 A-B & $07461+2107$ & $\mathrm{HO}$ & 247 & 37909 & 1.77 & 245.5 & 0.48 & 6.1 & 0.06 & Seymour \& Hartkopf (1999) \\
\hline $21276 \mathrm{~A}-\mathrm{B}$ & $07518-1354$ & $\mathrm{BU}$ & 101 & 38382 & 1.60 & 293.9 & 0.53 & -0.4 & -0.03 & Pourbaix (2000) \\
\hline 22187 A-B & $08061-0047$ & $\mathrm{~A}$ & 1971 & 39641 & 1.64 & 13.5 & 0.976 & 2.1 & 0.067 & Olević et al. (1993) \\
\hline 22565 A-B & $08122+1739$ & $\mathrm{STF}$ & 1196 & 40167 & 1.75 & 152.9 & 0.921 & -2.9 & 0.320 & Söderhjelm (1999) \\
\hline $25030 \mathrm{~A}-\mathrm{B}$ & $08554+7048$ & $\mathrm{STF}$ & 1280 & 43820 & 1.68 & 170.1 & 0.872 & -4.8 & -0.010 & Heintz (1997) \\
\hline 25914 A-B & $09144+5241$ & $\mathrm{STF}$ & 1321 & 120005 & 1.81 & 90.5 & 17.476 & 0.1 & 0.089 & Chang (1972) \\
\hline 26395 A-B & $09245+1808$ & A & 2477 & 46137 & 1.59 & 336.0 & 0.46 & -5.7 & 0.05 & Mason \& Hartkopf (1998) \\
\hline 28139 A-B & $10043-2823$ & I & 292 & 49336 & 1.72 & 325.3 & 0.46 & -1.6 & -0.03 & Seymour \& Mason (2000) \\
\hline 31184 A-B & $11182+3132$ & $\mathrm{STF}$ & 1523 & 55203 & 1.68 & 39.1 & 0.975 & 8.4 & 0.042 & Mason et al. (1995) \\
\hline 33231 A-B & $12160+0538$ & STF & 1621 & 59816 & 1.66 & 31.0 & 0.834 & 10.0 & -0.118 & Söderhjelm (1999) \\
\hline 33523 A-B & $12244+2535$ & STF & 1639 & 60525 & 1.75 & 326.8 & 1.621 & 1.3 & -0.022 & Olević \& Popović (2000) \\
\hline 33740 A-B & $12306+0943$ & STF & 1647 & 61035 & 1.59 & 243.9 & 1.359 & -1.1 & 0.057 & Hopmann (1970) \\
\hline 35047 A-B & $13100+1732$ & $\mathrm{STF}$ & 1728 & 64241 & 1.63 & 6.7 & 0.39 & -5.6 & -0.09 & Hartkopf et al. (1989) \\
\hline 37216 A-B & $14131+5520$ & $\mathrm{STF}$ & 1820 & 69442 & 1.67 & 114.0 & 2.579 & -0.5 & 0.057 & va et al. (1998) \\
\hline 38571 A-B & $14542-6625$ & HJ & 4707 & 72921 & 1.73 & 296.6 & 0.68 & -0.2 & -0.02 & Mason et al. (1999) \\
\hline 39584 A-B & $15232+3017$ & $\mathrm{STF}$ & 1937 & 75312 & 1.72 & 29.9 & 1.032 & -1.4 & -0.005 & Mason et al. (1999) \\
\hline 39999 A-B & $15360+3948$ & $\mathrm{STT}$ & 298 & 76382 & 1.67 & 342.5 & 0.38 & 2.6 & 0.04 & Söderhjelm (1999) \\
\hline 41102 A-B & $16044-1122$ & $\mathrm{STF}$ & 1998 & 78727 & 1.55 & 47.7 & 0.65 & -1.7 & 0.04 & Söderhjelm (1999) \\
\hline 41482 A-B & $16147+3352$ & $\mathrm{STF}$ & 2032 & 79607 & 1.54 & 234.6 & 6.79 & 0.1 & -0.06 & Scardia (1979) \\
\hline $41525 \mathrm{~A}-\mathrm{B}$ & $16160+0721$ & $\mathrm{STF}$ & 2026 & 79702 & 1.57 & 21.3 & 3.152 & 0.1 & 0.086 & Heintz (1963) \\
\hline 42506 A-B & $16413+3136$ & STF & 2084 & 81693 & 1.68 & 77.5 & 1.579 & 1.1 & 0.047 & Söderhjelm (1999) \\
\hline 43418 A-B & $17053+5428$ & STF & 2130 & 83608 & 1.58 & 30.4 & 2.161 & 1.7 & 0.040 & Heintz (1981) \\
\hline 43835 A-B & $17153-2636$ & SHJ & 243 & 84405 & 1.50 & 329.9 & 4.837 & 0.3 & 0.101 & Irwin et al. (1996) \\
\hline 44529 A-B & $17304-0104$ & $\mathrm{STF}$ & 2173 & 85667 & 1.53 & 332.2 & 1.107 & 0.2 & -0.006 & Heintz (1994) \\
\hline 44901 A-B & $17386+5546$ & $\mathrm{STF}$ & 2199 & 86344 & 1.75 & 60.0 & 1.939 & 0.9 & 0.056 & Popović \& Pavlović (1995) \\
\hline $48381 \mathrm{~A}-\mathrm{B}$ & $18432+5933$ & STF & 2398 & 91768 & 1.53 & 170.8 & 13.36 & 0.2 & 0.10 & Heintz (1987) \\
\hline $48456 \mathrm{C}-\mathrm{D}$ & $18443+3940$ & $\mathrm{STF}$ & 2383 & 91919 & 1.69 & 86.8 & 2.340 & 0.8 & 0.021 & Docobo \& Costa (1984) \\
\hline $50071 \mathrm{~A}-\mathrm{B}$ & $19121+4951$ & $\mathrm{STF}$ & 2486 & 94336 & 1.65 & 207.8 & 7.635 & 0.2 & -0.031 & Hale (1994) \\
\hline 50979 A-B & $19266+2719$ & $\mathrm{STF}$ & 2525 & 95589 & 1.62 & 291.8 & 1.963 & 0.2 & 0.028 & Heintz (1984) \\
\hline $51816 \mathrm{~A}-\mathrm{B}$ & $19394+2215$ & $\mathrm{STF}$ & 2556 & 96691 & 1.64 & 14.0 & 0.36 & -3.2 & 0.00 & Aristidi et al. (1999) \\
\hline 52207 A-B & $19450+4508$ & $\mathrm{STF}$ & 2579 & 97165 & 1.68 & 230.1 & 2.483 & 1.5 & -0.045 & Scardia (1983b) \\
\hline $56430 \mathrm{~A}-\mathrm{B}$ & $20462+1554$ & STF & 2725 & 102490 & 1.57 & 9.5 & 5.963 & -0.6 & -0.035 & Hopmann (1973) \\
\hline $56462 \mathrm{~A}-\mathrm{B}$ & $20467+1607$ & STF & 2727 & 102531 & 1.57 & 266.6 & 9.353 & 0.3 & -0.074 & Hale (1994) \\
\hline 57584 A-B & $21069+3845$ & STF & 2758 & 104214 & 1.69 & 148.0 & 30.063 & -0.4 & -0.135 & Kisselev et al. (1997) \\
\hline 59456 A-B & $21441+2845$ & $\mathrm{STF}$ & 2822 & 107310 & 1.97 & 302.6 & 1.990 & -0.5 & 0.018 & Heintz (1995) \\
\hline 61409 A-B & $22266-1645$ & SHJ & 345 & 110778 & 1.59 & 349.0 & 2.220 & -0.3 & 0.060 & Hale (1994) \\
\hline 62057 A-B & $22409+1433$ & $\mathrm{HO}$ & 296 & 111974 & 1.76 & 63.1 & 0.48 & 2.1 & 0.00 & Söderhjelm (1999) \\
\hline $65184 \mathrm{~A}-\mathrm{B}$ & $23595+3343$ & STF & 3050 & 118281 & 1.67 & 321.1 & 1.853 & 1.2 & 0.007 & Starikova (1977) \\
\hline
\end{tabular}

${ }^{1}$ Note that TDSC 227 A-B has two calibration quality orbits. This is a case where the ensemble of visual data does not show a clear preference of one orbit over the other, though the Tycho data here fit the Heintz (1993) orbit better. 


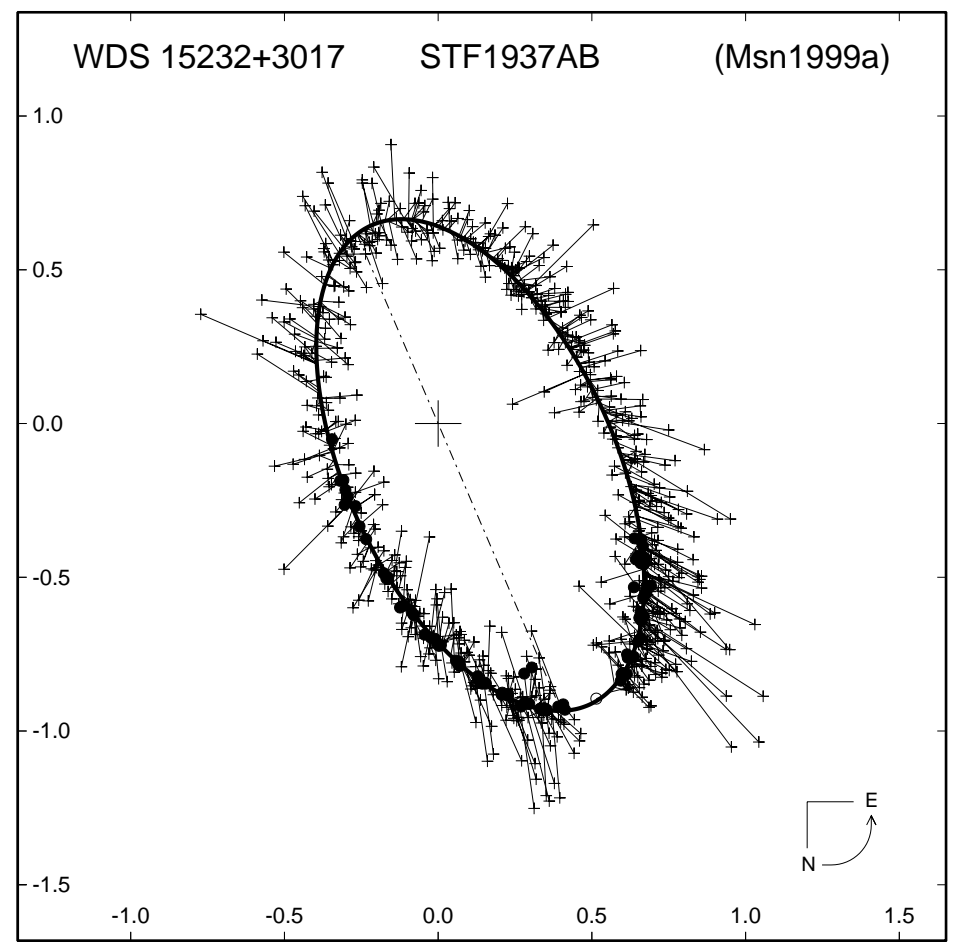

Fig. 5. The double star measures of TDSC 39584 (= STF1937AB = WDS 15232+3017) are plotted against the definitive orbit of Mason et al. (1999). Double star measures made with a micrometer are illustrated as plus signs while measures made by speckle interferometry are dots. The open circle at lower right is the measure of Tycho. For clarity, all other measures within 5 degrees in position angle of the Tycho measure are omitted. Data points are connected with their predicted positions on the orbit by $\mathrm{O}-\mathrm{C}$ lines. The broken line is the line of nodes and the axes of the figure are in arcseconds. The direction of motion is indicated in the lower right corner. Position angles are measured from north through east.

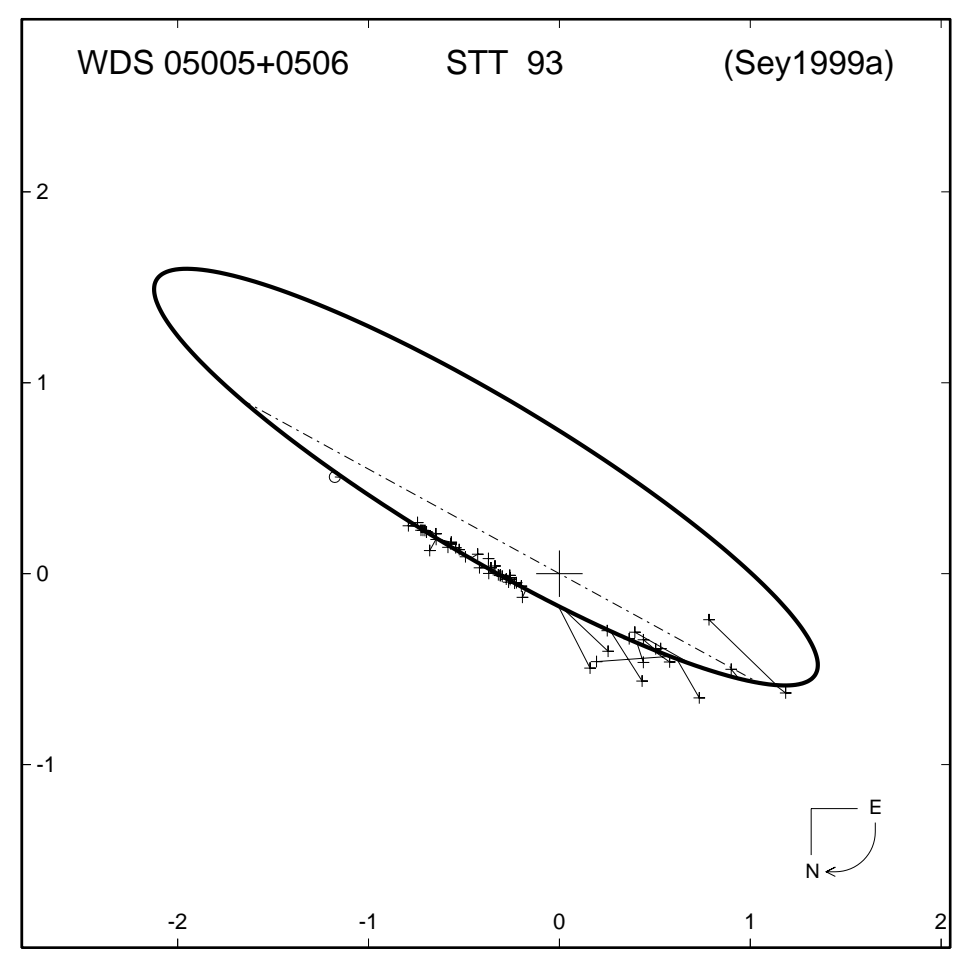

Fig. 6. The double star measures of TDSC 10552 (= STT $93=$ WDS 05005+0506) are plotted against the provisional (but still calibration grade) orbit of Seymour \& Mason (1999). All symbols are as Fig. 5.

\section{Description of the catalogue}

Section 3 describes the stellar contents of the catalogue. It consists of a main catalogue with 98482 entries and a supplement with 4777 entries, as detailed in Table 1, and is available from the astronomical data centres, 


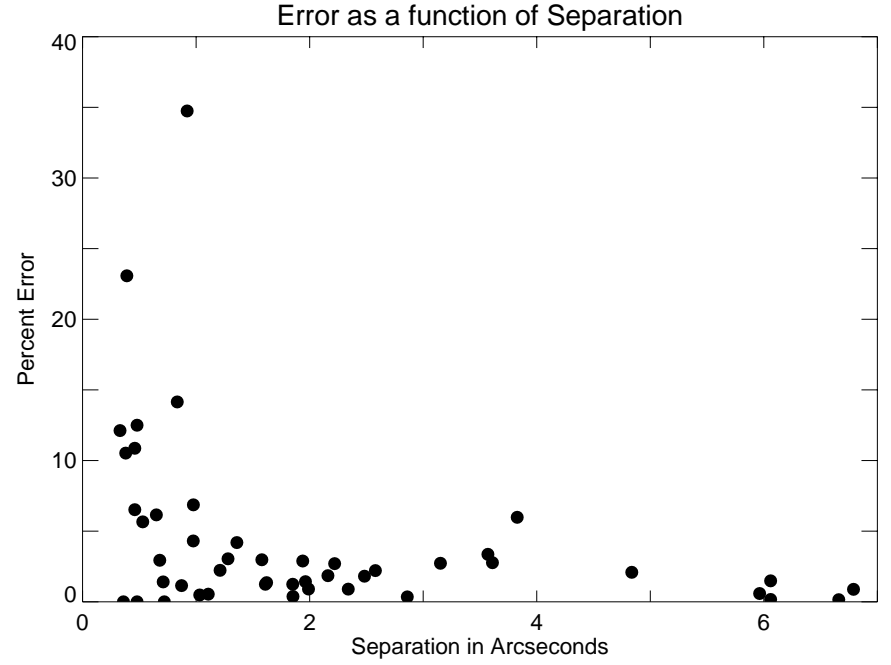

Fig. 7. Separation vs. $\%$ error in separation $\left(\frac{\left|(\mathrm{O}-\mathrm{C})_{\rho}\right|}{\rho}\right)$ for the closer systems listed in Table 2. At a little less than 1 arcsec the error drops to $\sim 10 \%$.

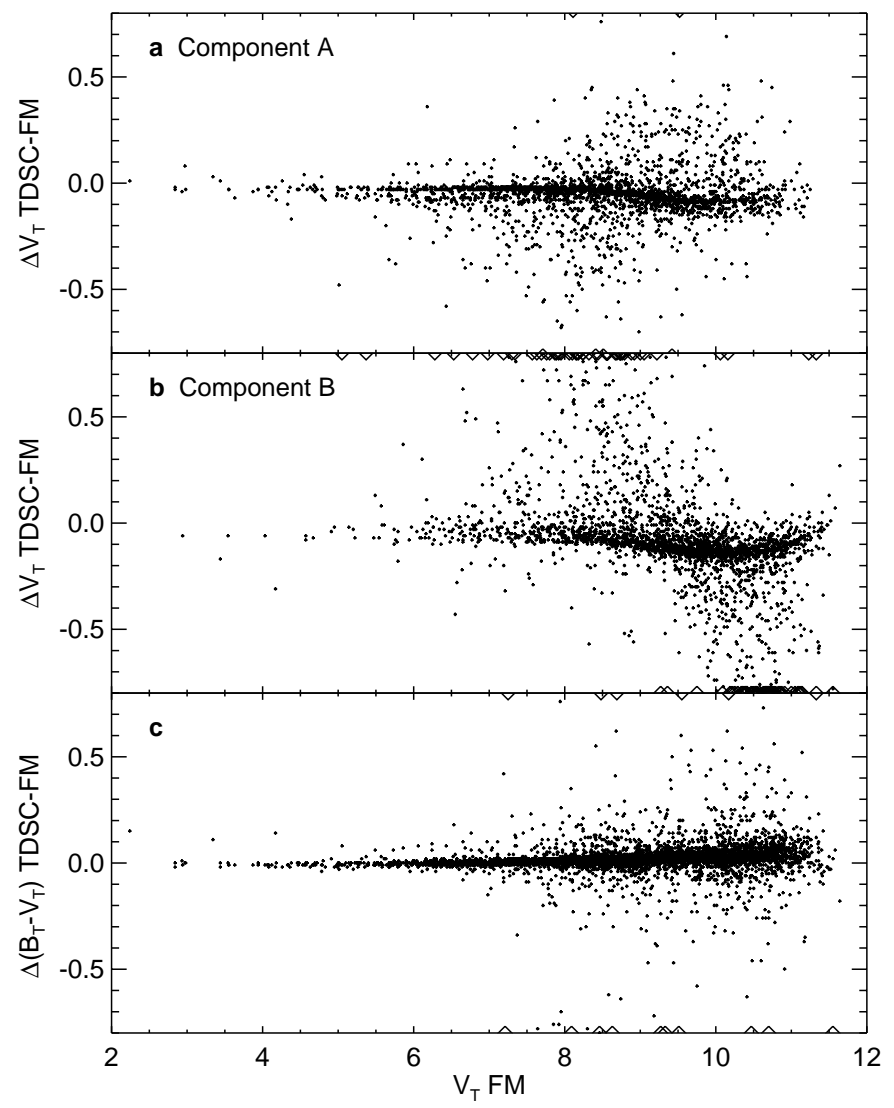

Fig. 8. Comparison of the TDSC photometry for about 2750 Hipparcos double stars, with supposedly more accurate photometry from Fabricius \& Makarov (2000a). Outliers are indicated with triangles.

accompanied by a detailed format description. Here we give only a summary of the contents.

- Running number, 1.665210, for the system. Please note that some systems are split between main

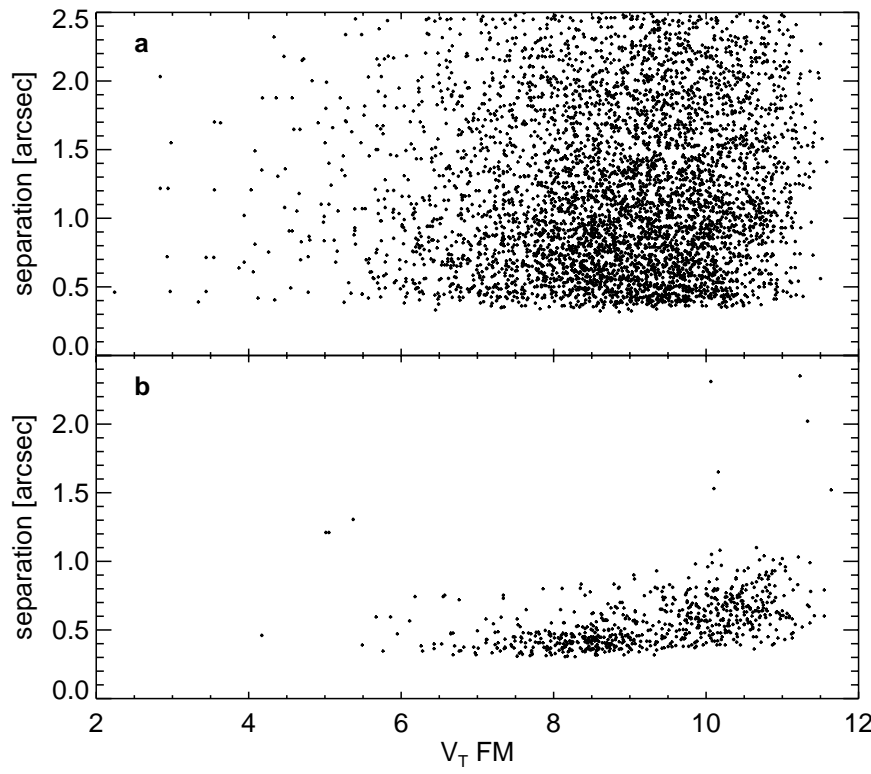

Fig. 9. The distribution of the stars from Fig. 8 with respect to magnitude and separation. Panel a) shows those with $V_{\mathrm{T}}$ differences below 0.25 , while panel $\mathbf{b}$ ) shows those with larger differences.

catalogue and supplement, and some are in fact only in the supplement;

- a unique component designation. As far as possible consistent with WDS designations;

- number of components, $0 \ldots 11$, in the main catalogue and in the supplement for the system;

- RA and Dec (ICRS) at the epoch of observation ( 1991.5). A flag indicates whether the position is from the new reduction $(50 \%)$ or is copied from Tycho-2 (50\%). For the supplement, the position is either copied from Tycho-1 (21\%) or copied from Hipparcos (79\%);

- proper motions. A flag indicates if it is copied from Tycho- 2 and valid for the photocentre $(47 \%)$ or valid for the individual component (49\%). For $4 \%$ no proper motion is available and for $0.03 \%$ it is copied from Hipparcos;

- $B_{\mathrm{T}}$ and $V_{\mathrm{T}}$ photometry is provided for all stars in the main catalogue and for many stars in the supplement. For the remaining stars the Hipparcos magnitude $(\mathrm{Hp})$ is given. A flag details the several possible sources of the photometry;

- Tycho-2 identification with new TYC3 numbers for the fainter component of newly resolved stars. Only three double stars are not in Tycho-2 at all, as explained in Sect. 3.1;

- cross identifications to WDS, Hipparcos (including component letters) and HD. The latter is taken from Fabricius et al. (2002);

- position angle and separation are provided relative to a previously listed component of the same system. They are computed for the mean observational epoch of the two stars. Position angles are given with respect to the ICRS pole; 
- a note is given for some stars to explain peculiarities, mostly due to complications with the WDS identification.

Acknowledgements. This work was supported by the Danish Space Board. Thanks are provided to the U.S. Naval Observatory for their continued support of the double star program.

\section{References}

van Albada, G. B. 1957, Cont. Bosscha Obs., 5

Aristidi, É., Prieur, J.-L., Scardia, M., et al. 1999, A\&AS, 134, 545

Chang, K. 1972, AJ, 77, 759

Docobo, J. A., \& Costa, J. M. 1984, IAU Circ. 92

Dommanget, J. 2000, Observatory, 120, 202

Dommanget, J., \& Nys, O. 1994, Comm. de l'Obs. R. de Belg. Ser. A, No. 115

ESA 1997, The Hipparcos and Tycho Catalogues, ESA SP-1200

Fabricius, C., \& Makarov, V. V. 2000a, A\&A, 356, 141

Fabricius, C., \& Makarov, V. V. 2000b, A\&AS, 144, 45

Fabricius, C., Makarov, V. V., Knude, J., \& Wycoff, G. L. 2002, submitted

Hale, A. 1994, AJ, 107, 306

Hartkopf, W. I., \& Mason, B. D. 2000, IAU Circ. 142

Hartkopf, W. I., McAlister, H. A., \& Franz, O. G. 1989, AJ, 98,1014

Hartkopf, W. I., Mason, B. D., \& Worley, C. E. 2001, AJ, 122, $3472^{2}$

Heintz, W. D. 1963, Veröff. München, 5, 247

Heintz, W. D. 1981, PASP, 93, 90

Heintz, W. D. 1984, A\&AS, 56, 5

Heintz, W. D. 1987, PASP, 99, 1084

Heintz, W. D. 1993, A\&AS, 98, 209

Heintz, W .D. 1994, AJ, 108, 2338

Heintz, W. D. 1995, ApJS, 99, 693

Heintz, W. D. 1997, ApJS, 111, 335

Heintz, W. D. 2001, IAU Circ., 143

Hopmann, J. 1970, Astron. Mitt. Wien, 5

Hopmann, J. 1973, Astron. Mitt. Wien, 13

Høg, E., Fabricius, C., Makarov, V. V., et al. 2000a, A\&A, 357, 367

Høg, E., Fabricius, C., Makarov, V. V., et al. 2000b, A\&A, 363, 385
Irwin, A. W., Yang, S. L. S., \& Walker, G. A. H. 1996, PASP, 108,580

Kisselev, A. A., Kiyaeva, O. V., \& Romanenko, L. G. 1997, in Visual Double Stars: Formation, Dynamics and Evolutionary Tracks, ed. J. A. Docobo, A. Elipe, \& H. McAlister, 377

Kiyaeva, O. V., Tokovinin, A. A., \& Kalinichenko, O. A. 1998, SvAL, 24, 753

Mason, B. D., \& Hartkopf, W. I. 1998, IAU Circ., 134

Mason, B. D., McAlister, H. A., Hartkopf, W. I., \& Shara, M. M. 1995, AJ, 109, 332

Mason, B. D., Douglass, G. G., \& Hartkopf, W. I. 1999, AJ, 117,1023

Mason, B. D., Hartkopf, W. I., Holdenried, E. R., et al. 2000a, AJ, 120,1120

Mason, B. D., Wycoff, G. L., Urban, S. E., et al. 2000b, AJ, 120,3244

Mason, B. D., Hartkopf, W. I., Wycoff, G. L., et al. 2001a, AJ, 122,1586

Mason, B. D., Wycoff, G. L., Hartkopf, W. I., Douglass, G. G., \& Worley, C. E. 2001b, AJ, 122, $3466^{3}$

Olević, D. J., \& Popović, G. M. 2000, IAU Circ. 141

Olević, D., Popović, G. M., Zulević, D. J., \& Ćatović, Z. 1993, Bull. Obs. Astron. Belgrade, 148, 49

Popović, G. M., \& Pavlović, R. 1995, Bull. Obs. Astron. Belgrade, 151, 45

Popović, G. M., \& Pavlović, R. 1996, Bull. Obs. Astron. Belgrade, 153, 57

Pourbaix, D. 2000, A\&AS, 145, 215

Scardia, M. 1979, AN, 300, 307

Scardia, M. 1983a, AN, 304, 257

Scardia, M. 1983b, IAU Circ. 89

Seymour, D. M., \& Hartkopf, W. I. 1999, IAU Circ. 139

Seymour, D. M., \& Mason, B. D. 1999, IAU Circ. 139

Seymour, D. M., \& Mason, B. D. 2000, IAU Circ. 140

Söderhjelm, S. 1999, A\&A, 341, 121

Starikova, G. A. 1977, Soobchen. Gos. Astr. Inst. Sternberga, 199, 12

Worley, C. E. 1981, in Observational Techniques and Results for Visual Double Stars in Current Techniques in Double and Multiple Star Research, IAU Colloq. 62, ed. R. S. Harrington, \& O. G. Franz, Lowell Observatory Bulletin, No. 167, vol. 9, No. 1,1 